\title{
Hexamethylenetetramine as a corrosion inhibitor in hydrochloric acid
} solution

\author{
Liujia1, a, He Daibin2,b, Zhang Mingjun²,c, Dong Juntao2,d and Xuchang1,e \\ ${ }^{1}$ Chongqing University Of Science and Technology, China \\ ${ }^{2}$ Southwest Oil and Gas Field Branch Shunan Gas Field, China \\ 42966338@qq.com, hhedabin@petrochina.com.cn, \\ 'Zhhangmingjun@petrochina.com.cn, ${ }^{\mathrm{d}}$ sndongit@petrochina.com.cn, \\ e454817480@qq.com
}

\begin{abstract}
Keywords: Hexamethylenetetramine(HMTA); inhibition corrosion efficiency; temperature; concentration; weight loss method

Abstract. The corrosion inhibition and absorption behavior of Hexamethylenetetramine(HMTA) for carbon steel in hydrochloric acid solution were studied by weight loss method. The results showed that as the temperature increases, the corrosion inhibition of HMTA effect after the first increased, reached the maximum at $30{ }^{\circ} \mathrm{C}$; With the increase of corrosion inhibitor concentration of HMTA, the concentration was $2.0 \%$, the efficiency of the corrosion in maximum, is greater than the concentration, the corrosion inhibition efficiency decline; In 15\% hydrochloric acid concentration, the corrosion effect is best.
\end{abstract}

\section{Introduction}

The formula for HMTA is $\left(\mathrm{CH}_{2}\right)_{6} \mathrm{~N}_{4}$, non-toxic, no smell, suitable for use in oil pipeline, steam boiler, hot water boiler in a common corrosion inhibitor. As the HTMA is often found in practical application corrosion phenomenon, leading to the corrosion effect exists question. HMTA and other material to compound with only have corrosion effect. HMTA only have corrosion in hydrochloric acid. There are different ideas about HMTA corrosion effect on temperature relations. In this paper, the effect of HMTA corrosion were discussed.

\section{Research on the corrosion mechanism of the HMTA}

The corrosion mechanism of HMTA research are two aspects of physical adsorption and chemical adsorption. Because HMTA can decompose the formaldehyde under acid condition, due to the effect of van der Waals force, has small molecular weight of formaldehyde adsorption on the metal surface, have the effect of corrosion. On the other hand, the $\mathrm{N}$ atoms in the HMTA, have 
unpaired electron pair, and metal surface has d empty orbits, they will form the coordination between health, which makes the HMTA adsorption in the form of chemical adsorption on the metal surface, have the effect of corrosion.

\section{The performance of the corrosion}

Static corrosion rate

According to the law of the People's Republic of China oil and gas industry standards SY/T5405-1996 'Acidification corrosion inhibitor performance test methods and evaluation indexes'. By using static coupon weight loss method, under normal pressure, corrosion temperature $90{ }^{\circ} \mathrm{C}$, the measuring of $\mathrm{N} 80$ steel in $1 \%$ corrosion inhibitor, industrial hydrochloric acid corrosion rate of $4 \mathrm{~h}$. Corrosion rate and corrosion inhibition rate respectively according to the type (1), (2) calculation.

$$
\begin{aligned}
V_{i} & =\frac{\Delta m_{i} \times 10^{6}}{A_{i} \times \Delta t} \\
\eta & =\frac{\left(V_{0}-V_{i}\right)}{V_{0}} \times 100 \%
\end{aligned}
$$

in this equation，错织!未找到引用漂。一 The corrosion rate of corrosion inhibitor single chip， $\mathrm{g} /\left(\mathrm{m}^{2} \cdot \mathrm{h}\right)$

$$
\begin{aligned}
& \Delta \mathrm{t} \text { - The corrosion reaction time, } \mathrm{h} ; \\
& \Delta m_{i}-\text { Sheet steel corrosion mass loss, g; } \\
& A_{i}-\text { Surface area of the steel, } \mathrm{mm}^{2} ; \\
& V_{0}-\text { Blank corrosion rate, } \mathrm{g} /\left(\mathrm{m}^{2} \cdot \mathrm{h}\right) 。
\end{aligned}
$$

The results and discussion

The influence of temperature on the corrosion rate

The N80 steel slice into the mixed with 15\% hydrochloric acid and 15\% HMTA solution (mass fraction, the same below), respectively in different temperature determine the efficiency of the corrosion test, the result is shown in figure 1.

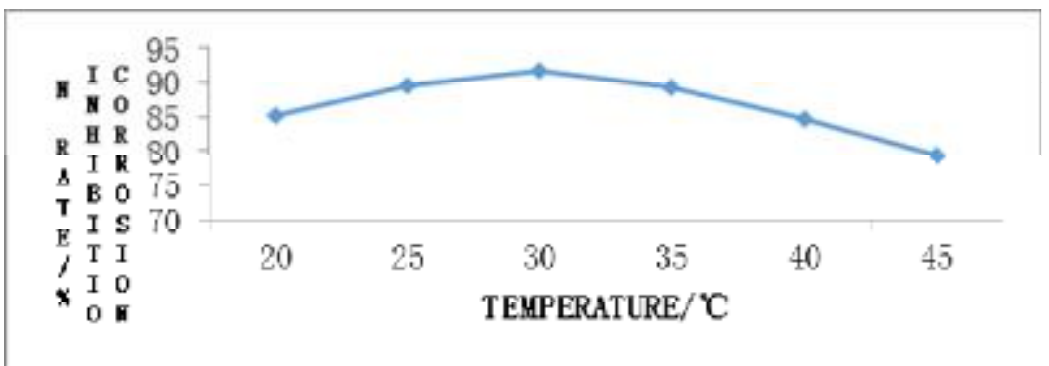

Fig 1 temperature effect on the rate of corrosion inhibitor 
With the increase of reaction temperature, the corrosion rate increases, arrived at $30{ }^{\circ} \mathrm{C}$ maximum corrosion rate, and then began to decrease. the efficiency of the corrosion inhibitor concentration

The added amount of HMTA in 15\% concentration of hydrochloric acid solution and the corrosion rate of $\mathrm{N} 80$ steel relations as shown in figure 2.

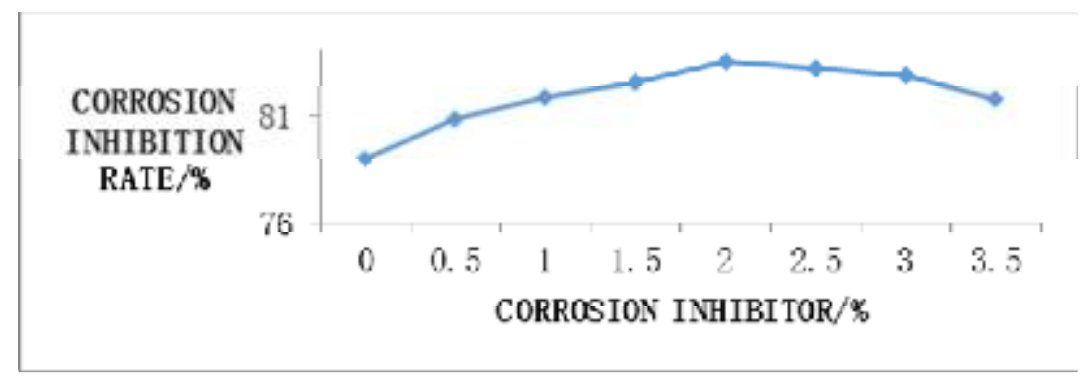

Fig 2 The amount of inhibitor on the corrosion effect

By shown in figure 2, with the increase of the concentration of the HMTA, the corrosion inhibitor concentration was $2.0 \%$, the efficiency of the corrosion in maximum, is greater than the concentration, the inhibition efficiency decline.

Then the effects of concentration of hydrochloric acid and the corrosion rate

Temperature setting at $30{ }^{\circ} \mathrm{C}$, the N80 steel slice into with different mass fraction of hydrochloric acid and $2.0 \%$ of the efficiency of corrosion in the HMTA in the mixed solution sample, the result is shown in figure 3.

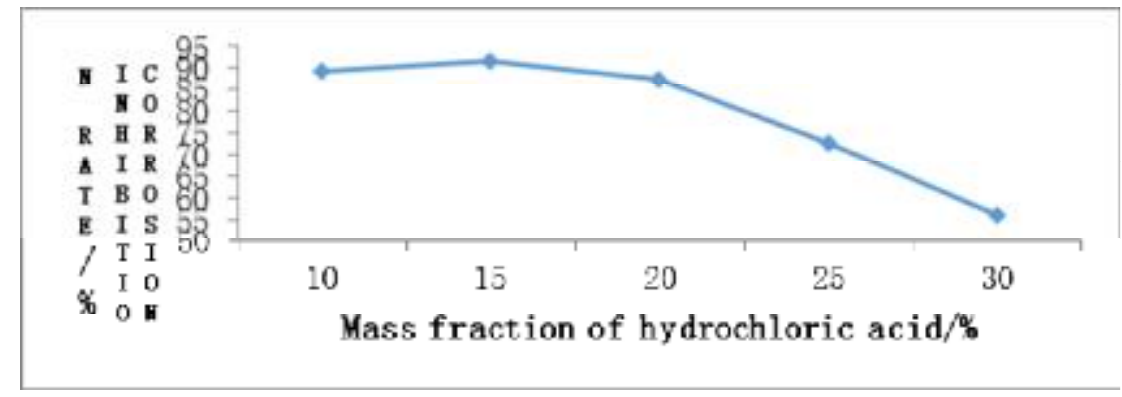

Fig.3 The efficiency of corrosion in the HMTA

With the increase of concentration of hydrochloric acid, the corrosion rate increased to $15 \%$ mass fraction of hydrochloric acid after began to fell sharply, with the increase of concentration of HMTA in the inhibitor concentration was $2.0 \%$, the efficiency of the corrosion in maximum, is greater than the concentration, the inhibition efficiency decline.

Discussion

The concentration of the HMTA at about $2.0 \%$ will achieve better corrosion effect, over this concentration range can aggravate corrosion effect instead. HMTA molecule has a large ring, in the iron adsorption on the surface, produce space steric effect in metal surface coverage is small. 
Consequently, the corrosion effect is not obvious, and the type of chair six-member ring, a single molecule of the coverage area is larger, so a small amount of HMTA concentration can achieve greater corrosion effect. With the increase of reaction temperature, the corrosion rate increases, arrived at $30{ }^{\circ} \mathrm{C}$ maximum corrosion rate, and then began to reduce. Internal heat of HMTA is relatively poor, when temperature increased acidification of formaldehyde volatilization, van der Waals force. In addition, the temperature led to increasing HMTA random movement, van der Waals force between the molecules is greater than $\mathrm{N}$ atoms and $\mathrm{d}$ empty orbital coordination of health, will reduce the efficiency of the corrosion. With the increase of concentration of hydrochloric acid, the corrosion rate in the mass fraction of hydrochloric acid began after fell sharply increased to $15 \%$.

\section{Conclusion}

(1)The temperature at $30{ }^{\circ} \mathrm{C}$, and the HMTA mass fraction in $2.0 \%, 15 \%$ for the corrosion inhibition of N80 steel in hydrochloric acid solution is the best.

(2)The experimental data show that when the HMTA mass fraction exceeds a certain value, reduce corrosion effects and has certain corrosion effect, at the same time temperature corrosion effect becomes poor.

\section{References}

[1] Wang Baocheng, Zhu Jinhua. Urotropine on the corrosion of steel in hydrochloric acid solution performance [J]. Journal of Beijing university of science and technology,2006,28(3):259- 262. [2] Zhou Qionghua, Yang Daowu, Zhu Zhipin. Tungstate and urotropine corrosion inhibition for carbon steel collaborative research [J]. North China electric power technology, 2004(3):3-5.

[3] Zhou Yong. Study of effects of corrosion and scaling in mixed acid cleaning liquid metal additives. [J]. Chemical engineer,2000, 79(4):44- 46.

[4] Zeng Rongchang, Han Enhou. The corrosion and protection materials [M]. Beijing: Chemical industry press,2006: 296.

[5]Gao Junlin。Urotropine as a corrosion inhibitor in hydrochloric acid solution [J].Corrosion and protection, 2009,3 (30) : 182-183. 